\title{
Telephone consultations in place of face to face out-patient consultations for patients discharged from hospital following surgery: a systematic review
}

\author{
Jo Thompson-Coon ${ }^{1 *}$, Abdul-Kareem Abdul-Rahman ${ }^{1}$, Rebecca Whear ${ }^{1}$, Alison Bethel ${ }^{1}$, Bijay Vaidya², \\ Christian A Gericke ${ }^{3,4}$ and Ken Stein ${ }^{1}$
}

\begin{abstract}
Background: Routine follow-up following uncomplicated surgery is being delivered by telephone in some settings. Telephone consultations may be preferable to patients and improve outpatient resource use. We aimed to compare the effectiveness of telephone consultations with face to face follow-up consultations, in patients discharged from hospital following surgery.

Methods: Seven electronic databases (including Medline, Embase and PsycINFO) were searched from inception to July 2011. Comparative studies of any design in which routine follow-up via telephone was compared with face to face consultation in patients discharged from hospital after surgery were included. Study selection, data extraction and quality appraisal were performed independently by two reviewers with consensus reached by discussion and involvement of a third reviewer where necessary.

Results: Five papers (four studies; 865 adults) met the inclusion criteria. The studies were of low methodological quality and reported dissimilar outcomes precluding any formal synthesis.

Conclusions: There has been very little comparative evaluation of different methods of routine follow-up care in patients discharged from hospital following surgery. Further work is needed to establish a role for telephone consultation in this patient group.
\end{abstract}

Keywords: Systematic review, Outpatient appointment scheduling, Surgery, Routine follow-up, Telephone consultation

\section{Background}

Rising costs and increasing demand for specialist services have increased interest in maximising the efficiency of managing specialist outpatient departments [1-8]. In this context, telephone follow-up consultations for various medical and surgical patient populations have been suggested as a means of improving the use of outpatient resources [9-12].

Providing routine follow-up by telephone may be considered preferable in situations where patients have to

\footnotetext{
* Correspondence: J.Thompson-Coon@exeter.ac.uk

'PenCLAHRC, University of Exeter Medical School, University of Exeter, Veysey Building, Salmon Pool Lane, Exeter EX2 4SF, UK

Full list of author information is available at the end of the article
}

travel from rural areas, and for those whose health or social conditions make hospital visits difficult [13]. Telephone consultations may also allow valuable outpatient clinic time to be occupied by those patients who have a more severe or complex conditions. This form of followup might also help improve compliance with scheduled follow-ups, avert the need for health care professionals to provide home visits, and prevent unnecessary return visits to the emergency department $[3,14]$.

However, it is also possible that telephone follow-up consultations may become an additional burden to the outpatient department, instead of reducing staff workloads with clinicians wary of missing or being unable to

\section{Biomed Central}

(c) 2013 Thompson-Coon et al.; licensee BioMed Central Ltd. This is an Open Access article distributed under the terms of the Creative Commons Attribution License (http://creativecommons.org/licenses/by/2.0), which permits unrestricted use, distribution, and reproduction in any medium, provided the original work is properly cited. 
confirm important clinical signs or other disease conditions $[9,15,16]$.

Anecdotal evidence suggests that surgical teams are routinely replacing face to face follow-up consultations with those made by telephone in some patient groups, where the intention is to discharge the patient from surgical specialist care. To date, there has been no synthesis of the evidence for the direct comparison between face to face follow-up consultations and those made by telephone in the context of surgical follow up care.

We therefore performed a systematic review to assess the effectiveness and, if possible, the cost effectiveness of telephone consultation as a method for routine followup consultations in secondary care for those patients who have undergone a surgical procedure, in comparison to (or as a replacement for) conducting follow-up appointments face to face.

\section{Methods}

The systematic review was conducted following the general principles published by the NHS Centre for Reviews and Dissemination (CRD) [17]. A pre-defined protocol was developed following consultation with topic and methods experts and is available from the PenCLAHRC website [http://clahrc-peninsula.nihr.ac. uk/est-projects.php].

\section{Literature search and eligibility criteria}

The search strategy was developed after consultation with clinical experts (RF, CG, BV) and examination of key papers. The search strategy for PsycINFO is shown below; the search was adapted to run in other databases. Studies were identified by searching the following electronic databases: Medline, Embase and PsycINFO (all via Ovid); AMED and CINAHL (both via NHS Evidence); and the Cochrane Library; and the Social Sciences Citation Index (in the Web of Science). These databases were searched from inception until July 2011. No search filters were applied. Due to resource constraints, only articles published in English were included. Reference lists of included papers were checked for further potentially relevant citations. The search was updated in June 2012.

\section{Search strategy for PsycINFO (OVID)}

Database: psycINFO <1806 to July Week 2 2012>

Search strategy:

1. exp surgery/ (33266)

2. exp Outpatients/ (4582)

3. exp telephone systems/ (2253)

4. exp clinics/ (4514)

5. exp Aftercare/ (912)

6. exp Hospital Discharge/ (2135)

7. 4 or 5 or 6 (7437)
8. 1 or $2(37824)$

9. 3 and 7 and 8 (4)

10. (surger* or surgi* or hospital* or outpatient* or operat*).tw. (225907)

11. (secondary adj care).tw. (476)

12. 10 or 11 (226244)

13. (tele ${ }^{*}$ or phone* or call? or calling).tw. (81736)

14. (appointment* or consult* or clinic* or \$discharge or review* or followup or aftercare).tw. (662394)

15. (follow adj up).tw. (63368)

16. 14 or 15 (698170)

17. (tele* or phone* or call? or calling).ti. (16117)

18. ((tele* or phone* or call? or calling) adj5 (appointment* or consult* or clinic* or \$discharge or review* ${ }^{*}$ or followup or aftercare)).tw. (2301)

19. 17 and 18 (881)

20. 14 and 17 (2600)

21.19 or 20 (2600)

22. 12 and 21 (409)

Studies were included if they compared the effects of telephone with face to face follow up consultations in patients who had undergone a surgical procedure in secondary care. Patients included in the studies had to be assessed as fit by their health care providers to be discharged to home and to receive a follow-up appointment in the surgical outpatient clinic. The telephone follow-up consultations needed to be planned and initiated by health care professionals and could be provided live or pre-recorded (or be automated). We excluded consultations in only text form. No limits on reported outcomes were imposed. Any study reporting comparative data was included irrespective of study design.

\section{Study selection}

Three authors (AA/JTC and RW) independently screened the titles and abstracts and applied the inclusion and exclusion criteria in a standardised and blinded manner. Duplicates were identified and discrepancies were reconciled through discussion. The full text of articles that were assessed as potentially meeting the inclusion criteria at this stage were retrieved and underwent the same process. Disagreements were arbitrated and resolved by discussion with others in the team (JTC and KS).

\section{Data extraction}

Data extraction was performed using a piloted data extraction form by one reviewer (AA/JTC or RW) and checked by a second (AA or RW). Disagreements were reconciled through discussion.

The following data were extracted for each study: 1) characteristics of the article (including author, title and year of the publication); 2) characteristics of the study participants (including mean age, underlying medical or 
surgical condition, the type surgical procedure performed, and inclusion and exclusion criteria); 3) characteristics of the study (such as the study design, sampling assumptions, whether the sample was derived randomly, the method of randomisation to intervention allocations, method of concealment, and method of blinding); 4) description of the intervention (who provided the telephone follow-up consultation, how soon after surgery it was carried out, and its duration); 5) descriptions of the comparator (including whether it was a face to face follow-up consultation, when it was provided, and duration); and 6) type of outcomes reported (including the variables reported, the values of the results, the standard deviations, and any statistical tests of association). The data extraction template is available from the authors on request.

\section{Assessment of quality and risk of bias}

Risk of bias was assessed using the principles published by the NHS Centre for Reviews and Dissemination [17], and the Cochrane Handbook of Systematic Reviews for Interventions [18]. One reviewer applied the criteria (AA/JTC or RW) which were checked by a second reviewer (AA/JTC or RW). Disagreements were resolved by discussion.

\section{Data synthesis}

Due to the heterogeneity of the study designs, the population and outcome measures reported in them, and the risk of bias identified, no formal statistical synthesis was performed. The results of the included studies were tabulated and evaluated in a narrative manner.

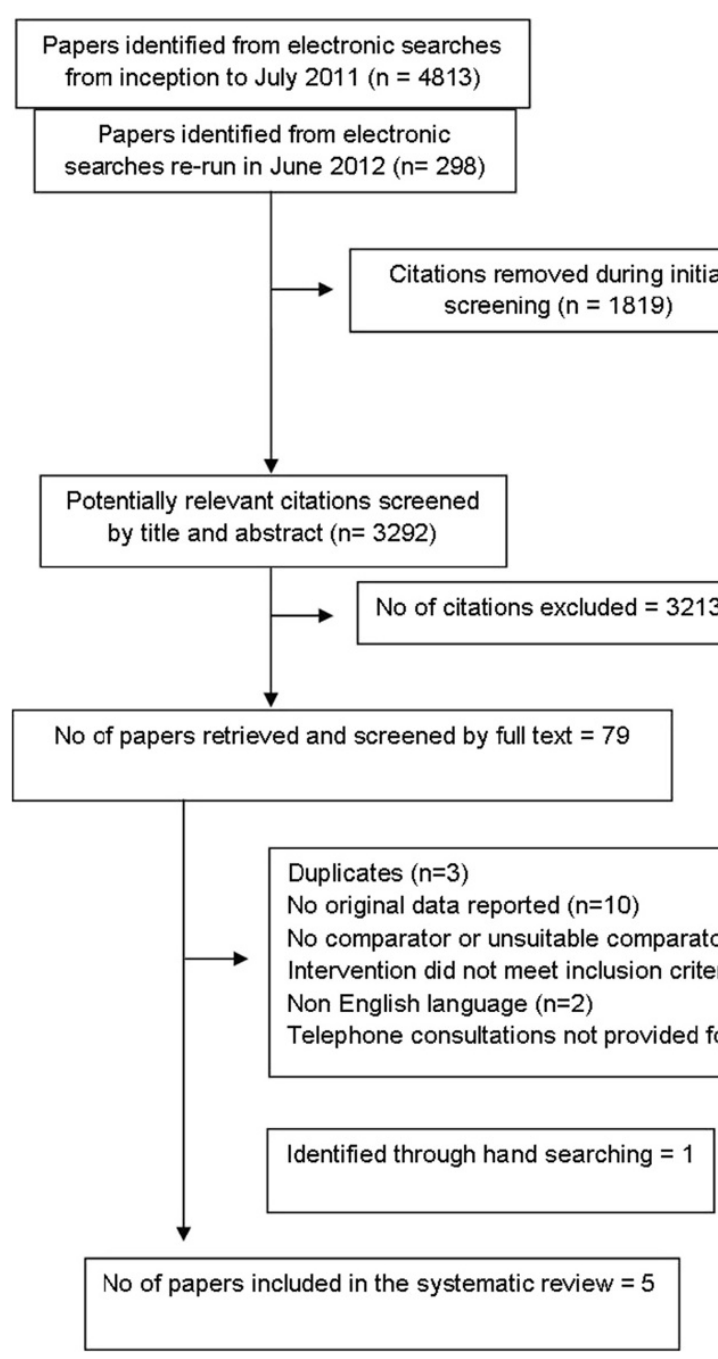

Figure 1 Process of study selection. 


\section{Results}

\section{Study selection}

A total of 4813 articles were identified by the initial search. Of these, 2,994 were unique (non duplicate) citations. The search was re-run in June 2012 and identified a further 298 unique references. Title and abstract screening resulted in the exclusion of 3213 citations. The full text of the remaining 79 articles was retrieved for further assessment. Seventy-five papers were excluded; three articles were duplicates, 10 were non empirical works, 33 did not include a comparator or the comparator was not face to face consultation in the outpatient department, in seven papers the intervention did not meet the inclusion criteria for the review and two papers were published in languages other than English. In 20 studies, the follow-up consultations were not provided directly following or in relation to surgery. One additional study was identified through hand searching. Five papers (four studies) were included in the review [19-23]. The process of study selection is summarised in Figure 1.

\section{Characteristics of included studies}

Two publications were concerned with post-operative care following dental work in maxillofacial surgery departments in the US; one a retrospective audit of patient records during a time period in which both telephone consultation and traditional outpatient follow-up strategies were in use [22] and the other an RCT in which patients were randomised to either method of follow-up [21]. One paper reports prospective data collected in a non-randomised trial on the post-operative experience following cataract surgery [23]. The other two publications report the findings of a single study in people undergoing nasal septal surgery in an otorhinolaryngology department in the UK $[19,20]$. The data for those undergoing telephone consultation follow-up in this study was collected prospectively and compared with historical records of patients seen traditionally in the outpatient department. In all studies the telephone consultation was standardised by the use of a proforma or questionnaire and was conducted at the same time point as the comparative clinic appointment. The characteristics of the included studies are summarised in Table 1. Sample size ranged from 48 in the RCT [21] to 364 in the retrospective audit of patient notes [22]; no sample size estimations were reported. Overall, the methodological quality of the included studies was poor with a moderate to high risk of bias for all results. Although described as a randomised trial, Sittitavornwong and colleagues [21] did not report any details of the process of randomisation or allocation concealment and the reporting of the results is very unclear. It is not clear how individuals were allocated to telephone or face to face follow-up in the studies by Susarla and colleagues [22] and Mandal and colleagues [23]. Retrospective sampling of patients over different time periods may subject the results to confounding influences which are not fully evident or reported. The methodological quality of each study is summarised in Table 2.

\section{Patient satisfaction outcomes}

Patient satisfaction has been measured in a number of ways across these four studies, precluding a simple summary of the findings. However, the results seem to suggest that patients were happy to receive routine followup care by telephone and in some cases showed a preference for this method. It is possible that the method used to administer the questionnaires e.g. face to face or over the telephone might have influenced the results; we were unable to look at this in detail due to the small number of included studies. In the study by Uppal and colleagues [19], a modified version of a validated outpatient satisfaction questionnaire was used; the language was modified slightly so that the same questionnaire could be used with both groups of patients. Patients were asked to respond on a five-point Likert scale (ranging from strongly agree to strongly disagree). The questions addressed issues such as whether the individual conducting the review was courteous and helpful and whether they listened to the patient. A total score was calculated for each patient and the results indicate that patient satisfaction was greater with nurse-led telephone follow-up than with conventional outpatient follow-up (mean difference in scores $0.39 ; 95 \%$ CI 0.17 to $0.61 ; \mathrm{p}=$ 0.001). Mandal and colleagues [23] used a non-validated questionnaire designed to assess the acceptability of telephone review as a post-operative assessment technique. The questions focussed on reassurance, comprehension and satisfaction with the service provided. There was no statistically significant difference in the proportion of patients saying that they were 'very reassured' or 'reassured' in the telephone review group compared with the eye infirmary clinic (although the difference between telephone review and review in the home visit group was significant). There were no significant differences in the level of understanding of post-operative materials between groups, nor in the assessment of the length of follow-up with all groups having a good level of comprehension and feeling that the length of the review was 'just right'. Although patients were asked which method of follow-up they preferred, their responses were not independent of their allocated group. More than 90\% of patients in the home visit group indicated a preference for a home visit and more than 70\% of the telephone group said that they preferred telephone follow-up. Sittitavornwong and colleagues [21] report that 73\% (35 of 48 patients) preferred telephone follow-up and $27 \%$ 
Table 1 Study characteristics

\begin{tabular}{|c|c|c|c|c|c|c|c|}
\hline Study, year & Design & Setting & Population & $\begin{array}{l}\text { Data } \\
\text { collection } \\
\text { periods }\end{array}$ & $\mathrm{n}$ & Intervention / comparator & Outcomes measured \\
\hline \multirow{4}{*}{$\begin{array}{l}\text { Uppal, } 2003[19] \\
\text { and Uppal, } 2004 \\
\text { [20] }\end{array}$} & \multirow{4}{*}{$\begin{array}{l}\text { Historically } \\
\text { controlled } \\
\text { trial }\end{array}$} & \multirow[t]{4}{*}{$\begin{array}{l}\text { Otolaryngology department; UK } \\
\text { hospital }\end{array}$} & \multirow[t]{3}{*}{$\begin{array}{l}\text { Patients undergoing nasal septal } \\
\text { surgery }\end{array}$} & \multirow[t]{3}{*}{$\begin{array}{l}\text { Dec } 2000 \\
\text { to Jan } 2002\end{array}$} & \multirow[t]{3}{*}{75} & \multirow[t]{3}{*}{$\begin{array}{l}\text { Telephone call with ENT nurse six weeks } \\
\text { after surgery using standardised protocol }\end{array}$} & $\begin{array}{l}\text { Patient satisfaction with } \\
\text { follow-up }\end{array}$ \\
\hline & & & & & & & Direct costs \\
\hline & & & & & & & Indirect costs \\
\hline & & & mean age 42 years; $76 \%$ male & $\begin{array}{l}\text { Jan } 1999 \text { to } \\
\text { Dec } 2000\end{array}$ & 78 & $\begin{array}{l}\text { Clinic appointment with surgeon (time } \\
\text { not specified) }\end{array}$ & Total costs of follow-up \\
\hline \multirow[t]{3}{*}{$\begin{array}{l}\text { Sittitavornwong, } \\
2005 \text { [21] }\end{array}$} & \multirow[t]{3}{*}{ RCT } & \multirow[t]{3}{*}{$\begin{array}{l}\text { Oral and maxillofacial surgery } \\
\text { department, US University }\end{array}$} & \multirow{2}{*}{$\begin{array}{l}\text { Patients undergoing third molar } \\
\text { removal performed under general } \\
\text { anaesthetic }\end{array}$} & \multirow[t]{3}{*}{$\begin{array}{l}\text { Not } \\
\text { reported }\end{array}$} & \multirow[t]{2}{*}{23} & \multirow[t]{2}{*}{$\begin{array}{l}\text { Telephone call at two weeks after } \\
\text { surgery using a questionnaire }\end{array}$} & $\begin{array}{l}\text { Patient satisfaction with } \\
\text { follow-up }\end{array}$ \\
\hline & & & & & & & Post-operative morbidity \\
\hline & & & mean age 20 years; $38 \%$ male & & 25 & Clinic appointment at two weeks & $\begin{array}{l}\text { Incidence of post-operative } \\
\text { help }\end{array}$ \\
\hline \multirow[t]{4}{*}{ Susarla, 2011 [22] } & \multirow[t]{4}{*}{$\begin{array}{l}\text { Retrospective } \\
\text { cohort }\end{array}$} & \multirow[t]{4}{*}{$\begin{array}{l}\text { Oral and maxillofacial surgery } \\
\text { department in a tertiary referral } \\
\text { centre, US }\end{array}$} & \multirow[t]{3}{*}{$\begin{array}{l}\text { Patients undergoing tooth } \\
\text { extraction in an ambulatory setting }\end{array}$} & \multirow[t]{4}{*}{$\begin{array}{l}\text { July } 2007 \\
\text { to June } \\
2009\end{array}$} & \multirow[t]{3}{*}{155} & \multirow[t]{3}{*}{$\begin{array}{l}\text { Telephone call with surgeon five to } \\
10 \text { days after surgery using a } \\
\text { standardised proforma }\end{array}$} & $\begin{array}{l}\text { Frequency of intra- } \\
\text { operative or postoperative } \\
\text { complication }\end{array}$ \\
\hline & & & & & & & Compliance with follow-up \\
\hline & & & & & & & $\begin{array}{l}\text { Patient satisfaction with } \\
\text { follow-up }\end{array}$ \\
\hline & & & mean age 28.6 years; $39 \%$ male & & 209 & $\begin{array}{l}\text { Clinic appointment with surgeon seven } \\
\text { to } 10 \text { days after surgery }\end{array}$ & No. of post-operative visits \\
\hline \multirow[t]{4}{*}{ Mandal, 2004 [23] } & \multirow{4}{*}{$\begin{array}{l}\text { Prospective } \\
\text { cohort }\end{array}$} & \multirow[t]{4}{*}{ Eye infirmary; UK hospital } & \multirow[t]{4}{*}{ Patients undergoing cataract surgery } & \multirow{4}{*}{$\begin{array}{l}\text { Not } \\
\text { reported }\end{array}$} & \multirow[t]{2}{*}{100} & \multirow{2}{*}{$\begin{array}{l}\text { Home visit by ophthalmic nurse one day } \\
\text { after surgery }\end{array}$} & Feelings of reassurance \\
\hline & & & & & & & $\begin{array}{l}\text { Level of understanding of } \\
\text { post-operative information }\end{array}$ \\
\hline & & & & & 100 & $\begin{array}{l}\text { Clinic appointment with ophthalmic } \\
\text { nurse one day after surgery }\end{array}$ & $\begin{array}{l}\text { Satisfaction with length of } \\
\text { review }\end{array}$ \\
\hline & & & & & 100 & $\begin{array}{l}\text { Telephone call using a structured review } \\
\text { form one day after surgery }\end{array}$ & \\
\hline
\end{tabular}




\section{Table 2 Indicators of study quality}

\begin{tabular}{|c|c|c|c|c|}
\hline Quality indicator & Uppal, 2003 [19]; Uppal, 2004 [20] & Sittitavornwong, 2005 [21] & Susarla, 2011 [22] & Mandal, 2004 [23] \\
\hline Was the objective of the study clearly described? & Yes & Yes & Yes & Yes \\
\hline Are the main outcomes to be measured clearly described? & Yes & Yes & Yes & Yes \\
\hline Are the characteristics of the included subjects clearly described? & Yes & No & Partial & No \\
\hline Was there a clearly defined point in time when the intervention occurred? & Yes & Yes & Yes & No \\
\hline Are the interventions of interest clearly described? & Yes & No & Yes & Yes \\
\hline Are possible confounders clearly described? & Partial & Partial & Partial & No \\
\hline Are the main findings of the study clearly described? & Yes & Yes & Yes & Yes \\
\hline Are estimates of the random variability in the data provided? & Yes & Yes & Yes & No \\
\hline Are all adverse events that may be a consequence of the intervention reported? & Unclear & Unclear & Unclear & Unclear \\
\hline Are the characteristics of patients lost to follow-up described? & NA & Yes & NA & No \\
\hline Were losses of patients to follow up accounted for? & NA & Yes & NA & No \\
\hline Are the actual probability values reported for the main outcomes? & Yes & No & Yes & No \\
\hline Were attempts made to blind outcome assessors to the intervention? & NA & No & NA & NA \\
\hline Were the groups recruited from the same population? & No & Yes & Yes & Yes \\
\hline Were the groups recruited over the same period of time? & No & Yes & Yes & Unclear \\
\hline Was a representative sample of the population approached? & Yes & Yes & Unclear & Unclear \\
\hline Was the intervention unlikely to affect data collection? & Yes & Yes & Yes & Yes \\
\hline Was there reliable compliance with the intervention? & Partial & Yes & NA & Yes \\
\hline Were the main outcome measures used valid and reliable? & Yes & Yes & Yes & No \\
\hline Was the study free from selective outcome reporting? & Unclear & Unclear & Unclear & Unclear \\
\hline Were baseline characteristics similar between groups? & Unclear & Unclear & Unclear & Unclear \\
\hline
\end{tabular}


(13 of 48) preferred clinic follow-up. These results are difficult to interpret since they provide information on the proportion of the total number of patients in the study $(n=48)$ reporting these preferences, rather than the proportion per randomised group. Susarla and colleagues asked patients if they were satisfied with the telephone post-operative follow-up compared with a clinic visit and the answer was recorded as 'yes' or 'no' [22].

\section{Costs}

Two studies report costs [20,23]. Uppal and colleagues in 2004 estimate the direct and indirect health care related costs of telephone follow-up consultations at $£ 50$ per patient, compared to $£ 106.11$ for those conducted face to face [20]. A simple cost calculation in the paper by Mandal and colleagues estimates the cost of a telephone review at between $£ 1$ and $£ 2$; it is not clear which costs have been included in these calculations and there is no information provided about the cost of an outpatient appointment in the eye infirmary (the estimated cost of a home visit was £8.50) [23].

\section{Frequency of complications}

The data provided by Sittitavornwong and colleagues is difficult to interpret since they provide information on the proportion of the total number of patients in the study experiencing complications rather than the number per randomised group [21] (Table 3). In the study by Susarla and colleagues [22], there were significantly more complications in the group receiving telephone review. However, when the results were adjusted to account for differences in the two groups, no statistically significant difference was seen.

\section{Discussion}

Despite conducting a widespread search for possible data in a variety of sources we were unable to identify any good quality comparative evidence for the use of telephone consultations in place of face to face outpatient appointments after surgery. Although from a limited pool of surgical modalities, data from all four studies suggests that patients were happy to receive routine follow-up care by telephone and in most instances showed some preference to this method compared with an appointment in the outpatient department.

However, the methodological quality of all the identified studies was poor with a moderate to high risk of bias for all the results. In addition, the studies were small, patient satisfaction was measured in a variety of ways and there is little information available on longterm outcomes. The limitations of the included studies have consequences for any sensible extrapolation of the results to other clinical situations.
This research question arose following discussion with surgeons in our local hospital who are routinely replacing face to face outpatient appointments with telephone calls following some surgical procedures. In an environment of increasing cost and demand and a need for ever greater efficiency, it is disappointing that we were able to identify so little comparative research.

In this review, we have taken considerable steps to identify all relevant published and unpublished papers by searching seven electronic databases and hand searching the reference lists of all included papers and many others identified during the search. Although we were only able to include papers published in the English language due to resource constraint, which may have resulted in the omission of some evidence, our searches were not limited to English only and did not identify a significant body of literature in other languages. Very few studies have compared the methods of care and as far as we are aware, this is the first systematic review to specifically address the effectiveness and acceptability of telephone consultation in place of face to face outpatient appointments in patients following uncomplicated surgery.

The authors of a Cochrane Review of telephone consultations in the three months following discharge from hospital identified 33 papers in which telephone followup consultations had been evaluated following a wide range of treatments in many patient groups, using a range of comparators and using a variety of outcome measures [24]. In most cases there was no statistically significant difference between telephone and control groups and no meta-analysis was possible. None of the studies identified adverse effects of telephone follow-up. Our review takes a more focused approach than the previous work, addressing explicitly the use of telephone follow up after surgery and compared this with face-to -face hospital based appointments (not home visits). None of the studies included in our review were identified in the Cochrane Review. Furthermore, we have looked at complications related to the surgical operation as this goes to the issue of whether telephone consultations in this context are safe - a necessary pre-requisite for their implementation as routine practice.

Post-surgical consultations may be used for the exchange of a variety of different kinds of information e.g. education, identification of complications, reassurance, management of pain and symptoms. Moreover, the aim for consultations, in most cases, will be discharge from specialist care. It is therefore necessary that the consultation takes careful account of all possible harms and patient concerns. Telephone consultation have been studied in only a small number of conditions and, while they appear to be an innovative use of health service resources, uncertainty remains about their effects and whether these are consistent in different conditions. 
Table 3 Study results

\begin{tabular}{|c|c|c|c|c|c|c|c|c|c|}
\hline \multirow[t]{2}{*}{ Study, year } & \multicolumn{2}{|c|}{ Uppal, 2003 [19]; Uppal, 2004 [20] } & \multicolumn{2}{|c|}{ Sittitavornwong, 2005 [21] } & \multicolumn{2}{|c|}{ Susarla, 2011 [22] } & \multicolumn{3}{|c|}{ Mandal, 2004 [23] } \\
\hline & Telephone & Face to face & Telephone & Face to face & Telephone & Face to face & Telephone & Home visit & Face to Face \\
\hline$n$ & 75 & 75 & 25 & 23 & 155 & 209 & $100^{\$}$ & $100^{\$}$ & $100^{\$}$ \\
\hline \multicolumn{10}{|l|}{ Cost outcomes } \\
\hline Total cost of follow-up ( $(£)$ & 3760.88 & 7958.25 & - & - & - & - & - & - & - \\
\hline Cost per patient $(£)$ & 50.15 & 106.11 & - & - & - & - & 1 to 2 & 8.50 & - \\
\hline \multicolumn{10}{|l|}{ Patient satisfaction outcomes } \\
\hline Patient satisfaction with follow-up & $1.04(0.50)^{* \S}$ & $0.65(0.52) * \S$ & - & - & $95.9 \%^{\dagger}$ & - & - & - & - \\
\hline Proportion reporting 'very reassured' & - & - & - & - & - & - & $69.6 \%$ & $83.9 \%$ & $79.5 \%$ \\
\hline Proportion showing good level of understanding & - & - & - & - & - & - & $84 \%$ & $93.1 \%$ & $87.2 \%$ \\
\hline Patient preference for follow-up method & - & - & $73 \% \%^{* *}$ & $27 \% * *$ & & & - & - & - \\
\hline Satisfaction with length of review & - & - & - & - & - & - & \multicolumn{3}{|c|}{$97.2 \%$ just right } \\
\hline Compliance with follow-up & $83 \%$ & $93 \%$ & $83 \%$ & $77 \%$ & $97.1 \%$ & $94.5 \%$ & - & - & - \\
\hline \multicolumn{10}{|l|}{ Frequency of complications } \\
\hline Frequency of intra-operative or post-operative complication & - & - & & & $12.9 \%{ }^{\#}$ & $23.4 \%^{\#}$ & - & - & - \\
\hline on day one & & & $19 \%(n=9)^{* *}$ & $10 \%(n=5)^{* *}$ & & & & & \\
\hline at two weeks & & & $10 \%(n=5)^{* *}$ & $2 \%(n=1)^{* *}$ & & & & & \\
\hline Incidence of post-operative help & - & - & $8 \%(n=4)^{* *}$ & $6 \%(n=3)^{* *}$ & & & - & - & - \\
\hline
\end{tabular}

${ }^{*}$ mean (sd); ${ }^{5}$ missing data for 33 patients in the telephone group and 29 patients in the face to face group; ${ }^{* *}$ these data are reported as a proportion of 48 patients;

${ }^{+}$self reported patient satisfaction rate in $82 \%$ of patients; ${ }^{*}$ after adjusting for differences between the samples, no significant difference was found in complication frequencies ( $\left.p=0.7\right)$; ${ }^{5}$ only $82 \%$ of patients completed or partially completed questionnaires; - not reported. 
There is therefore a need for good quality prospective and, crucially, comparative evaluations of telephone consultations with outpatient appointments in post-surgical care which seek to collect information on all aspects of care. Whilst telephone consultations might be more convenient for patients, there is no indication from the published literature whether this type of after-care has any impact on the frequency of adverse events.

\section{Conclusion}

There has been very little comparative evaluation of different methods of routine follow-up care in patients discharged from hospital following surgery. Further rigorous work is needed to establish a role for telephone consultation in this patient group as a replacement for face to face follow up in hospital outpatient departments.

\section{Competing interests}

The authors declare that they have no competing interests.

\section{Authors' contributions}

JTC was substantially involved in the conception and design of this review as well as data analysis and interpretation. JTC was also involved in writing and revising the manuscript and has approved this final version. AA was substantially involved in the conception and design of this review as well as data analysis and interpretation. AA was also involved in writing the manuscript and has approved this final version. RW was substantially involved in the conception and design of this review as well as data analysis and interpretation. RW was also involved in revising the manuscript and has approved this final version. $A B$ was substantially involved in running the literature searches, managing the databases and retrieving articles. $A B$ was also involved in revising the manuscript and has approved this final version. BV was substantially involved in the conception and design of this review. BV was also involved in revising the manuscript and has approved this final version. CG was involved in the conception and design of this review and was also involved in revising the manuscript and has approved this final version. KS was substantially involved in the conception and design of this review as well as its interpretation. KS was also involved in revising the manuscript and has approved this final version. All authors read and approved the final manuscript.

\section{Acknowledgments}

The authors are grateful to Professor Robert Freeman (Plymouth Hospitals NHS Trust and University of Plymouth) for useful discussions during the development of the protocol.

\section{Funding}

This systematic review is an independent research project funded by National Institute for Health Research (NIHR) Collaboration for Leadership in Applied Health Research and Care for the South West (PenCLAHRC). The views expressed in this publication are those of the authors and not necessarily those of the NHS, the NIHR, or the Department of Health.

\footnotetext{
Author details

${ }^{1}$ PenCLAHRC, University of Exeter Medical School, University of Exeter, Veysey Building, Salmon Pool Lane, Exeter EX2 4SF, UK. ²Department of Endocrinology, Royal Devon \& Exeter Hospital NHS Foundation Trust, Exeter EX2 5DW, UK. ${ }^{3}$ PenCLAHRC, National Institute for Health Research, Peninsula College of Medicine and Dentistry, University of Plymouth, Portland Square, Plymouth PL4 8AA, UK. ${ }^{4}$ The Wesley Research Institute and Queenland University of Technology, Brisbane, QLD 4068, Australia.
}

\section{References}

1. Hennessy D, Connolly S, Lennon G, Quinlan D, Mulvin D: Out-patient management and non-attendance in the current economic climate. How best to manage our resources? Ir Med J 2010, 103(3):80-82.

2. Dechartres A, Mazeau V, Grenier-Sennelier C, Brezin AP, Vidal-Trecan GM: Improving the organization of consultation departments in university hospitals. J Eval Clin Pract 2007, 13(6):930-934.

3. Bromage SJ, Napier-Hemy RD, Payne SR, Pearce I: Outpatient follow up appointments; are we using the resources effectively? Postgrad Med J 2006, 82(969):465-467.

4. Bratlid D: Lack of patients? A hypothesis for understanding discrepancies between hospital resources and productivity. BMC Health Serv Res 2006, 6:42.

5. Dunnill MG, Pounder RE: Medical outpatients: changes that can benefit patients. Clin Med 2004, 4(1):45-49.

6. Saine PJ, Baker SM: What is the best way to schedule patient follow-up appointments? Jt Comm J Qual Saf 2003, 29(6):309-315.

7. Hughes ML, Leslie SJ, Mclnnes GK, McCormac K, Peden NR: Can we see more outpatients without more doctors? J R Soc Med 2003, 96(7):333-337.

8. Wilson NV, Collins RE: Surgical outpatient practices-a time for change? Ann R Coll Surg Engl 1994, 76(4 Suppl):169-171.

9. Car J, Sheikh A: Telephone consultations. BMJ 2003, 326(7396):966-969.

10. Cusack M, Taylor C: A literature review of the potential of telephone follow-up in colorectal cancer. J Clin Nurs 2010, 19(17-18):2394-2405.

11. Cox K, Wilson E: Follow-up for people with cancer: nurse-led services and telephone interventions. J Adv Nurs 2003, 43(1):51-61.

12. McBride CM, Rimer BK: Using the telephone to improve health behavior and health service delivery. Patient Educ Couns 1999, 37(1):3-18.

13. Gray RT, Sut MK, Badger SA, Harvey CF: Post-operative telephone review is cost-effective and acceptable to patients. Ulster Med J 2010, 79(2):76-79.

14. Christensen AA, Lugo RA, Yamashiro DK: The effect of confirmation calls on appointment-keeping behavior of patients in a children's hospital dental clinic. Pediatr Dent 2001, 23(6):495-498.

15. Hannis MD, Hazard RL, Rothschild M, Elnicki DM, Keyserling TC, DeVellis RF: Physician attitudes regarding telephone medicine. J Gen Intern Med 1996, 11(11):678-683.

16. Welch HG, Johnson DJ, Edson R: Telephone care as an adjunct to routine medical follow-up. A negative randomized trial. Eff Clin Pract 2000, 3(3):123-130.

17. Centre for Reviews and Dissemination: Systematic Reviews: CRD's guidance for undertaking reviews in health care. York: Centre for Reviews and Dissemination, University of York; 2009.

18. Higgins JPT, Altman DG: Assessing Risk of Bias in Included Studies. In Cochrane Handbook for Systematic Reviews of Interventions. London: John Wiley \& Sons, Ltd; 2008:187.

19. Uppal S, Nadig S, Mielcarek MW, Smith L, Jose J, Coatesworth AP: Patient satisfaction with conventional and nurse-led telephone follow-up after nasal septal surgery. Int J Clin Pract 2003, 57(9):835-839.

20. Uppal S, Nadig S, Smith L, Coatesworth AP: A cost-effectiveness analysis of conventional and nurse-led telephone follow-up after nasal septal surgery. Ann R Coll Surg Engl 2004, 86(4):243-246.

21. Sittitavornwong S, Waite PD, Holmes JD, Klapow JC: The necessity of routine clinic follow-up visits after third molar removal. $J$ Oral Maxillofac Surg 2005, 63(9):1278-1282.

22. Susarla SM, Black R, TB D: After dentoalveolar surgery, most patients are satisfied with telephone follow-up. J Oral Maxillofac Surg 2011, 69(8):2099-2105.

23. Mandal K, Dodds SG, Hildreth A, Fraser SG, DHW S: Comparative study of first-day postoperative cataract review methods. J Cataract Refract Surg 2004, 30(9):1966-1971.

24. Mistiaen P, Poot E: Telephone follow-up, initiated by a hospital-based health professional, for postdischarge problems in patients discharged from hospital to home. Cochrane Database Syst Rev 2008, 3, CD004510.

\section{doi:10.1186/1472-6963-13-128}

Cite this article as: Thompson-Coon et al:: Telephone consultations in place of face to face out-patient consultations for patients discharged from hospital following surgery: a systematic review. BMC Health Services Research 2013 13:128. 